\title{
CONSISTENT STABLE DIFFERENCE SCHEMES FOR NONLINEAR BLACK-SCHOLES EQUATIONS MODELLING OPTION PRICING WITH TRANSACTION COSTS*
}

\author{
RAFAel COMPANY ${ }^{1}$, LuCAS JÓdAR ${ }^{1}$ And José-Ramón Pintos ${ }^{1}$
}

\begin{abstract}
This paper deals with the numerical solution of nonlinear Black-Scholes equation modeling European vanilla call option pricing under transaction costs. Using an explicit finite difference scheme consistent with the partial differential equation valuation problem, a sufficient condition for the stability of the solution is given in terms of the stepsize discretization variables and the parameter measuring the transaction costs. This stability condition is linked to some properties of the numerical approximation of the Gamma of the option, previously obtained. Results are illustrated with numerical examples.
\end{abstract}

Mathematics Subject Classification. 35K55, 65M12, 39A10, 90A09.

Received August 1st, 2008. Revised February 25, 2009.

Published online June 12, 2009.

\section{INTRODUCTION}

It is well-known that Black-Scholes (B-S) model is acceptable in idealized financial markets where one assumes that volatility is observable or transaction costs are not taken into account.

These unrealistic assumptions have been shown too restrictive in practice and this fact motivates the search of alternative models expressed by parabolic diffusion-convection equations for the option value $V$ as a function of the underlying security $S$ and the time

$$
V_{t}+\frac{1}{2}\left(\sigma\left(S, t, V_{S}, V_{S S}\right)\right)^{2} S^{2} V_{S S}+r S V_{S}-r V=0, S>0, t \in[0, T[
$$

with final and boundary conditions. Here $r \geq 0$ denotes the riskless interest rate and $\sigma$ means the volatility. Equations of this type arise frequently in mathematical finance, not only in option pricing, for instance in the modeling of transaction costs [2,5], optimal portfolios in incomplete markets [13] and inverse problems [9].

For the particular case where the volatility function $\sigma$ appearing in (1.1) is a constant, the model (1.1) turns out the B-S. In the B-S model for the pricing of options the influence of transaction costs was neglected and it was possible to construct a riskless portfolio that perfectly replicates the option pay-off. If transaction costs

\footnotetext{
Keywords and phrases. Nonlinear Black-Scholes equation, option pricing, numerical analysis, transaction costs.

* This paper has been partially supported by the Spanish Ministry of Education and Research grant TRA2007-68006-C02-02, the Generalitat Valenciana grant GVPRE/2008/092 and the Universidad Politécnica de Valencia grant 20070307.

1 Instituto Universitario de Matemática Multidisciplinar, Universidad Politécnica de Valencia, Edificio 8G, piso 2, P.O. Box 46022, Valencia, Spain. rcompany@imm.upv.es; ljodar@imm.upv.es; jrpt60@gmail.com
} 
are taken into account perfect replication of the contingent claim is no longer possible and it has been shown in [18] that further restrictions have to be imposed in the model.

If bid-ask spreads and other transaction costs are also taken into account, each adjustment of the portfolio implies an additional cost and the replication property of the B-S hedge no longer holds. Making frequent adjustments to maintain the theoretical hedge can increase costs considerably. On the other hand, if only a few adjustments are made, the B-S exact hedge cannot be maintained due to movements in the price of the underlying asset between trades. Therefore, transaction costs cannot be ignored without incurring risk or loss. This is an important practice problem, especially in emerging markets where round-trip transaction costs of $1 \%$ and higher are not uncommon.

There are two competing approaches behind modeling transaction costs. One is the so called local in time methods [21] characterized by the assumption of hedging strategies taking place at discrete times. The second approach based on the work [10], the so called global in time methods, characterized by the use of optimality techniques and utility functions. Practical drawbacks of this last approach have motivated the search of asymptotic techniques to derive manageable option pricing formulae.

Starting with the local in time methods, Leland [14] introduced a theory for pricing a call option with transaction costs showing that the price of a call is given by the B-S formula with an augmented volatility

$$
\sigma_{A}=\sigma_{0} \sqrt{1+A}, \quad A=\sqrt{\frac{2}{\pi}} \cdot \frac{k}{\sigma_{0} \sqrt{\triangle t}} .
$$

Here, $\sigma_{0}$ represents the volatility of the underlying security, $k$ is the round-trip transaction cost (a percentage) and $\triangle t$ is the time interval between successive adjustments of the portfolio. This time interval is considered fixed and is assumed to be much smaller than the time-to-expiration. We shall refer to the parameter $A$ as the Leland number.

Boyle and Vorst [3] derived a similar option pricing formula using a binomial model. Both approaches are restricted to derivative securities with convex payoffs.

In practice, there are many derivative securities of interest which have non-convex payoffs. The foremost example is a portfolio of standard options combining short and long positions. In [11] Hoggard et al. proposed an extension of Leland's result which applies to derivative securities with arbitrary payoff functions. Their model involves the solution of an equation of type (1.1) where

$$
\sigma=\sigma\left(V_{S S}\right)=\sigma_{0} \sqrt{1+A \operatorname{sign}\left(V_{S S}\right)} .
$$

Note that if $A \geq 1$, then by (1.3), $\sigma^{2}=\sigma_{0}^{2}\left(1+A \operatorname{sign}\left(V_{S S}\right)\right)$ is nonpositive if $V_{S S}<0$ which is unrealistic and ill-posed mathematically. From (1.2), $A \geq 1$ occurs when $\Delta t$ is enough small or $k$ is enough big. Avellaneda and Parás in [1] obtain the adjusted volatility (1.3) by proposing new hedging strategies to control effectively the hedging risk and transaction costs.

The global in time methods pioneered by Hodges and Neuberger [10] and developed by Davis et al. [5] achieve an element of optimality, since they are based on the approach of utility maximization. Since there are no explicit solutions for the utility based hedging with transaction costs and the numerical methods are computationally hard, for practical applications a suitable alternative is the use of an asymptotic solution. In asymptotic analysis one studies the solution to a problem when some parameters in the problem assume large or small values.

Whalley and Wilmott [21] were the first to provide an asymptotic analysis of the model of Hodges and Neuberger, assuming that transaction costs are small. Barles and Soner [2] performed an alternative method assuming that both the transaction costs and the hedger's risk tolerance are small. While Whalley and Wilmott derive only an optimal form of the hedging bandwith which is centered about the B-S delta, Barles and Soner show a particular form of the hedging bandwith and provide a nonlinear adjusted volatility of the form

$$
\sigma^{2}=\sigma_{0}^{2}\left(1+\Psi\left[\exp \left(r(T-t) a^{2} S^{2} V_{S S}\right)\right]\right),
$$


where $\mu$ is the proportional transaction cost; $a=\mu \sqrt{\gamma N}$, with risk aversion factor $\gamma$ and the number $N$ of options to be sold. The transaction costs increases with the higher values of $a$. When $a=0$, there is no transaction cost and classical Black-Scholes equation is recovered. The volatility correction function $\Psi$ is the solution of the nonlinear initial-value problem

$$
\Psi^{\prime}(A)=\frac{\Psi(A)+1}{2 \sqrt{A \Psi(A)}-A}, A \neq 0, \Psi(0)=0
$$

In the mathematical literature, only a few results can be found on the numerical discretization of B-S equation, mainly for linear B-S equations. The numerical approaches vary from finite element discretizations $[8,15]$, to finite difference approximations [6]. The numerical discretization of the B-S equation with the nonlinear volatility (1.3) has been performed using explicit finite-difference schemes in [1] and with the volatility given by (1.4) in [2]. However, explicit schemes have the disadvantage that restrictive conditions on the discretization parameters (for instance, the ratio of the time and the space step) are needed in order to obtain stable, convergent schemes [19]. [7] combines high-order compact difference schemes derived by [16] and techniques to construct numerical solutions with frozen values of the nonlinear coefficient of the non-linear B-S equation to make the formulation linear. Reasonable numerical strategies like the consideration of more discretization nodes near the maturity and the strike price, are not sufficient to guarantee reliability and accuracy of the numerical approximations. Careless numerical computations may waste a good mathematical model.

In this paper we deal with an European vanilla call option pricing equation (1.1) where $\sigma$ is given by (1.4)-(1.5), together with final and boundary conditions taking the form

$$
\left.\begin{array}{c}
V_{t}+\frac{1}{2} \sigma_{0}^{2}\left(1+\Psi\left[\mathrm{e}^{r(T-t)} a^{2} S^{2} V_{S S}\right]\right) S^{2} V_{S S}+r S V_{S}-r V=0, S>0, t \in[0, T[, \\
V(S, T)=\max (0, S-E), S>0, \\
V(0, t)=0, \lim _{s \rightarrow \infty} \frac{V(S, t)}{S-E \mathrm{e}^{-r(T-t)}}=1 .
\end{array}\right\}
$$

In [4] a semidiscretization technique is used to compute numerical solutions of problem (1.6) but no analysis of the numerical solution is developed.

From both the computational point of view and the numerical analysis, it is convenient the transformation of problem (1.6) into another simpler nonlinear parabolic problem.

In this paper we use an explicit finite difference scheme applied to the transformed problem. This scheme no only has the advantage that not uses frozen values of nonlinearities, such as in [7], but also guarantees that no spurious oscillations appear under certain stability condition previously determined.

In order to compute the numerical solution, it is necessary to work in a bounded domain. Once this numerical domain has been chosen, the boundary conditions of the continuous problem can be translated from the asymptotic condition to the boundary of the numerical domain, as it is done for instance in [2] or [7], or the boundary values of the numerical domain must be found together with the solution and they are linked with the rest of the numerical solution in the interior of the numerical domain by using extrapolation techniques. Here we use the second approach to keep the approximation order of the scheme.

This paper is organized as follows. In Section 2, the original problem (1.6) is transformed into a simpler nonlinear parabolic problem using appropriate change of variables. Some growth properties of the volatility correction function $\Psi$ defined by (1.5) are derived from an implicit formula. The function $g(A)=A \Psi(A)$ plays an important role in the numerical treatment of the problem because leads the behavior of the nonlinear term of both the original and the transformed equation. Smoothness properties of function $g(A)$ are also obtained in this Section 2. In Section 3, the finite-difference scheme is introduced for the computation of the numerical solution of the transformed problem. The scheme is first order forward in time $\tau$ and second order centered in the space variable $X$. Since suitable monotonic properties of the numerical solution of the problem are linked 
with the behaviour of the numerical approximation of the Gamma of the option $\frac{\partial^{2} V}{\partial S^{2}}$, a numerical scheme for this approximation of the Gamma is also introduced in Section 3. Section 4 gives a sufficient condition between the stepsize variables in order to guarantee the monotonic behaviour of the numerical solution of the problem with respect to the space variable at each time stage. This property means that the price of the European call option increases with the values of the asset variable, at every fixed time, as it is shown in Section 5, and it also guarantees the stability of the numerical solution. Section 5 includes the consistency of the numerical scheme with the equation of the transformed problem. Illustrative examples of results of previous sections are included in Section 6. Finally, references are mentioned.

\section{TRANSFormation of THE PROBlem AND PROPERTIES OF $\Psi$}

For the sake of convenience the PDE (1.1) is going to be transformed into a nonlinear diffusion equation. Although the authors of [2] do not use such a change to study the PDE (1.6), in some way it is suggested by them in [2], p. 379. Let us consider the substitution defined by

$$
X=\mathrm{e}^{r(T-t)} S ; \quad \tau=\frac{\sigma_{0}^{2}}{2}(T-t) ; \quad U=\mathrm{e}^{r(T-t)} V
$$

Hence,

and

$$
S=\mathrm{e}^{-2 \rho \tau} X ; \quad t=T-\frac{2 \tau}{\sigma_{0}^{2}} ; \quad V=\mathrm{e}^{-2 \rho \tau} U ; \quad \rho=\frac{r}{\sigma_{0}^{2}}
$$

$$
\begin{gathered}
\frac{\partial V}{\partial t}=\mathrm{e}^{-2 \rho \tau}\left(r U-\frac{\sigma_{0}^{2}}{2} \frac{\partial U}{\partial \tau}-r X \frac{\partial U}{\partial X}\right), \\
\frac{\partial V}{\partial S}=\frac{\partial U}{\partial X}, \quad \frac{\partial^{2} V}{\partial S^{2}}=\mathrm{e}^{r(T-t)} \frac{\partial^{2} U}{\partial X^{2}} .
\end{gathered}
$$

From (1.6) and (2.1)-(2.4) one gets

$$
L(U)=\frac{\partial U}{\partial \tau}-\left(1+\Psi\left(a^{2} X^{2} \frac{\partial^{2} U}{\partial X^{2}}\right)\right) X^{2} \frac{\partial^{2} U}{\partial X^{2}}=0, \quad 0<X<\infty, \quad 0<\tau \leq \frac{\sigma_{0}^{2} T}{2}
$$

together with the boundary conditions

$$
U(0, \tau)=0 ; \quad \lim _{X \rightarrow+\infty} U(X, \tau)=X-E,
$$

and the initial condition

$$
U(X, 0)=\max (0, X-E) .
$$

Dealing with numerical analysis of difference schemes presented in the next section, is going to be convenient to bound the approximation of the nonlinear term $\Psi\left(a^{2} X^{2} \frac{\partial^{2} U}{\partial X^{2}}\right)$ appearing in (2.5).

From Theorem 1.1 of [4] it is known that $\Psi(A)$ is an increasing function mapping the real line onto the interval $]-1,+\infty[$ and $\Psi(A)$ is implicitly defined by

$$
\begin{gathered}
A=\left(-\frac{\operatorname{Arcsinh} \sqrt{\Psi}}{\sqrt{\Psi+1}}+\sqrt{\Psi}\right)^{2}, \text { if } \Psi>0, \\
A=-\left(\frac{\arcsin \sqrt{(-\Psi)}}{\sqrt{\Psi+1}}-\sqrt{-\Psi}\right)^{2}, \text { if }-1<\Psi<0 .
\end{gathered}
$$


From (2.8), taking two times derivatives of the inverse function $A(\Psi)$ it is easy to check that

$$
\frac{\mathrm{d}^{2} A}{\mathrm{~d} \Psi^{2}}>0 \text { if } \Psi>0
$$

and

$$
\frac{\mathrm{d}^{2} \Psi}{\mathrm{d} A^{2}}=-\left(\frac{\mathrm{d}^{2} A}{\mathrm{~d} \Psi^{2}}\right)\left(\frac{\mathrm{d} \Psi}{\mathrm{d} A}\right)^{3}<0, \quad \text { if } \quad A>0 .
$$

Hence $\Psi(A)$ is a convex function for $A>0$. As $\lim _{A \rightarrow \infty} \frac{\Psi(A)}{A}=1$ see [2], p. 377, and from the convexity of $\Psi(A)$, taking

$$
\begin{aligned}
& A_{2}=\left(\sinh 2-\frac{2}{\sqrt{(\sinh 2)^{2}+1}}\right)^{2} \simeq 9.58, \Psi\left(A_{2}\right)=(\sinh 2)^{2}, \\
& \Psi^{\prime}\left(A_{2}\right)=\frac{\left(\mathrm{e}^{8}+2 \mathrm{e}^{4}+1\right)^{2}}{\mathrm{e}^{16}-66 \mathrm{e}^{8}+1} \approx 1.10, d_{2}=\Psi\left(A_{2}\right)-\Psi^{\prime}\left(A_{2}\right) A_{2} \simeq 2.62,
\end{aligned}
$$

one gets the upper bound

$$
0<\Psi(A) \leq \Psi^{\prime}\left(A_{2}\right) A+d_{2}, A>0 .
$$

The next lemma will play an important role in Section 5 to study the consistency of a numerical scheme.

Lemma 2.1. Let $\Psi(A)$ be the volatility correction function appearing in (1.6) verifying equation (1.5) and let us consider $g(A)=A \Psi(A)$. Then $g(A)$ is continuously differentiable at $A=0$ and satisfies

$$
\left|g^{\prime}(A)\right| \leq \max \left\{G, 2|A| \Psi^{\prime}\left(A_{2}\right)+d_{2}\right\}, \quad A \in \mathbb{R}
$$

where $A_{2}$ and $d_{2}$ are given by (2.10),

$$
A_{1}=-\frac{(4 \pi-3 \sqrt{3})^{2}}{36} ; \quad G=\max \left\{\left|g^{\prime}(A)\right| ; \quad A_{1} \leq A \leq A_{2}\right\}
$$

Proof. From (2.9), taking two times derivatives of the inverse function $A(\Psi)$ one gets

and

$$
\frac{\mathrm{d}^{2} A}{\mathrm{~d} \Psi^{2}}<0, \quad-1<\Psi<0,
$$

$$
\frac{\mathrm{d}^{2} \Psi}{\mathrm{d} A^{2}}=-\left(\frac{\mathrm{d}^{2} A}{\mathrm{~d} \Psi^{2}}\right)\left(\frac{\mathrm{d} \Psi}{\mathrm{d} A}\right)^{3}>0, \quad A<0 .
$$

Hence $\Psi(A)$ is concave in the domain $A<0$ and as $\lim _{A \rightarrow-\infty} \Psi(A)=-1$ from the concavity of $\Psi(A)$ for $A<0$, taking $A_{1}$ defined in (2.13) and $d_{1}=\Psi^{\prime}\left(A_{1}\right) A_{1}-\Psi\left(A_{1}\right) \simeq 0.64$ one gets

$$
|\Psi(A)| \leq \Psi^{\prime}\left(A_{1}\right)|A|+d_{1}, \quad A<0
$$

Let us take the Taylor expansions

$$
\left.\begin{array}{rl}
\operatorname{Arcsinh} x+x \sqrt{x^{2}+1} & =2 x+O\left(x^{2}\right), \\
-\operatorname{Arcsinh} x+x \sqrt{x^{2}+1} & =\frac{2 x^{3}}{3}+O\left(x^{4}\right), \\
\arcsin x+x \sqrt{1-x^{2}} & =2 x+O\left(x^{2}\right), \\
\arcsin x-x \sqrt{1-x^{2}} & =\frac{2 x^{3}}{3}+O\left(x^{4}\right) .
\end{array}\right\}
$$


Using (2.8), (2.9) together with (2.15) for both $x=\sqrt{\Psi}$ and $x=\sqrt{-\Psi}$ one gets the left-hand and right-hand derivatives

$$
\lim _{A \rightarrow 0^{ \pm}} g^{\prime}(A)=\lim _{A \rightarrow 0^{ \pm}} A \Psi^{\prime}(A)=\lim _{\Psi \rightarrow 0^{ \pm}} \frac{A(\Psi)}{A^{\prime}(\Psi)}=0 .
$$

Hence,

Otherwise

$$
\lim _{A \rightarrow 0} g^{\prime}(A)=0 .
$$

$$
g^{\prime}(0)=\lim _{A \rightarrow 0} \frac{g(A)-g(0)}{A}=\lim _{A \rightarrow 0} \frac{A \Psi(A)}{A}=\Psi(0)=0 .
$$

From (2.17)-(2.18) one gets that $g(A)$ is continuously differentiable at $A=0$.

Since $\Psi(A)$ is increasing positive and concave for $A>0$, from (2.11) one gets

$$
0<g^{\prime}(A)=\Psi(A)+A \Psi^{\prime}(A) \leq 2 A \Psi^{\prime}\left(A_{2}\right)+d_{2}, \quad A>A_{2} .
$$

Since $\Psi(A)$ is increasing negative and convex for $A<0$, and (2.14) one gets

$$
\left|g^{\prime}(A)\right| \leq|\Psi(A)|+|A|\left|\Psi^{\prime}(A)\right| \leq 2|A| \Psi^{\prime}\left(A_{1}\right)+d_{1}, \quad A<A_{1} .
$$

As $d_{2}>d_{1}>0$ and $\Psi^{\prime}\left(A_{2}\right)>\Psi^{\prime}\left(A_{1}\right)>0$, from (2.19), (2.20) is clear that

$$
\left.\left|g^{\prime}(A)\right| \leq 2|A| \Psi^{\prime}\left(A_{2}\right)+d_{2}, \quad A \in\right]-\infty, A_{1}[\cup] A_{2},+\infty[.
$$

From definition of $G$ given by (2.13) and (2.21) one gets (2.12).

\section{Numerical SCHEME CONSTRUCTION}

The bounded numerical domain can be chosen according with different criteria, but taking into account [12] the interval $[0,2 E]$ for the spatial variable $X$ related to the underlying asset variable $S$ is appropriate.

In order to construct numerical solutions of problem $(2.5)-(2.7)$, let us consider the bounded numerical domain $[0,2 E] \times\left[0, \frac{\sigma_{0}^{2} T}{2}\right]$ and the approximation of the partial derivatives $\frac{\partial^{2} U}{\partial X^{2}}$ and $\frac{\partial U}{\partial \tau}$ at the mesh points $\left(X_{j}, \tau^{n}\right)$, with $X_{j}=j(\triangle X)=j h ; \tau^{n}=n(\triangle \tau)=n k, 0 \leq j \leq N, 0 \leq n \leq l$, such that $N$ is even and

$$
\begin{gathered}
N h=2 E, \quad l k=\tau, \\
\frac{\partial^{2} U}{\partial X^{2}}\left(X_{j}, \tau^{n}\right)=\triangle_{j}^{n}+O\left(h^{2}\right), \\
\triangle_{j}^{n}=\triangle_{j}^{n}(u)=\frac{u_{j-1}^{n}-2 u_{j}^{n}+u_{j+1}^{n}}{h^{2}}, \quad 1 \leq j \leq N-1, \\
\frac{\partial U}{\partial \tau}\left(X_{j}, \tau^{n}\right)=\frac{u_{j}^{n+1}-u_{j}^{n}}{k}+O(k), \quad 0 \leq j \leq N,
\end{gathered}
$$

where $u_{j}^{n}$ denotes the approximation of the exact value of the solution of $(2.5)$ at $\left(X_{j}, \tau^{n}\right)$. Disregarding the errors in (3.2), (3.4) and substituting in (2.5) one gets the following scheme at the internal mesh points

$$
\begin{gathered}
u_{j}^{n+1}=\left(1-\frac{2 k}{h^{2}} \beta_{j}^{n}\right) u_{j}^{n}+\frac{k}{h^{2}} \beta_{j}^{n}\left(u_{j-1}^{n}+u_{j+1}^{n}\right), \quad 1 \leq j \leq N-1,0 \leq n \leq l-1 \\
\beta_{j}^{n}=\left(1+\Psi_{j}^{n}\right) X_{j}^{2}, \quad \Psi_{j}^{n}=\Psi\left(a^{2} X_{j}^{2} \triangle_{j}^{n}\right) .
\end{gathered}
$$


Remark 3.1. Note that from (3.6) and that $\Psi$ takes values in the interval $]-1,+\infty[$, see Theorem 1.1 of [4], the coefficients are nonnegative, $\beta_{j}^{n} \geq 0$.

With respect to the selection of the boundary values $u_{0}^{n}, u_{N}^{n}$ at the boundary of the numerical domain there are two mainly approaches. One of them, using for instance by Düring et al. in [7] translate the limit boundary condition (2.6) at the boundary of the numerical domain.

For problem (2.5)-(2.6), the translation of the boundary conditions to the boundary of the numerical domain takes the form

$$
u_{0}^{n}=0 ; \quad u_{N}^{n}=X_{N}-E=E, \quad 0 \leq n \leq l .
$$

Another method computes the values at the numerical domain boundaries as a part of the solution [20], p. 63. For this "purpose" they appear two artificial external mesh points $X_{-1}=-h, X_{N+1}=(N+1) h$ for which the values $u_{-1}^{n}$ and $u_{N+1}^{n}$ are assigned using Lagrange interpolation of an appropriate degree passing throughout the closest internal mesh points.

Approximation (3.2) suggests that linear Lagrange interpolation is sufficient in this case, taking

$$
u_{-1}^{n}=2 u_{0}^{n}-u_{1}^{n} ; \quad u_{N+1}^{n}=2 u_{N}^{n}-u_{N-1}^{n}, \quad 0 \leq n \leq l .
$$

Note that using scheme (3.5) for $j=0$, and the extrapolation conditions (3.8) one gets

$$
u_{0}^{n+1}=\left(1-\frac{2 k}{h^{2}} \beta_{0}^{n}\right) u_{0}^{n}+\frac{k}{h^{2}} \beta_{0}^{n}\left(u_{-1}^{n}+u_{1}^{n}\right)=u_{0}^{n}, \quad 0 \leq n \leq l-1 .
$$

In an analogous way, for $j=N$, one gets

$$
u_{N}^{n+1}=u_{N}^{n}, \quad 0 \leq n \leq l-1 .
$$

Since from the initial condition (2.7) $u_{0}^{0}=0$ and $u_{N}^{0}=X_{N}-E=E$, using (3.9) and (3.10) it follows that both treatment of the boundaries give the same values at the numerical boundary at each stage $n$.

Let us denote the vector $u^{n}=\left[u_{0}^{n}, u_{1}^{n}, \ldots, u_{N}^{n}\right]^{t}$ and let $A, B(n)$ be the matrices in $\mathbb{R}^{(N+1) \times(N+1)}$ defined by

$$
\begin{gathered}
A=\left[\begin{array}{ccccccc}
0 & 0 & 0 & 0 & 0 & \cdots & 0 \\
1 & -2 & 1 & 0 & 0 & \cdots & 0 \\
0 & 1 & -2 & 1 & 0 & \cdots & 0 \\
& \ddots & \ddots & \ddots & \ddots & \ddots & \\
0 & \cdots & 0 & 1 & -2 & 1 & 0 \\
0 & \cdots & 0 & 0 & 1 & -2 & 1 \\
0 & \cdots & 0 & 0 & 0 & 0 & 0
\end{array}\right], \\
B(n)=\operatorname{diag}\left(\beta_{0}^{n}, \beta_{1}^{n}, \ldots, \beta_{N}^{n}\right),
\end{gathered}
$$

where $\beta_{j}^{n}$ are defined like (3.6) also for $j=0, j=N$, and taking into account from (3.3) and (3.8) that

$$
\triangle_{0}^{n}=\triangle_{N}^{n}=0, \quad 0 \leq n \leq l-1,
$$

one gets

$$
\beta_{0}^{n}=0, \quad \beta_{N}^{n}=X_{N}^{2}, \quad 0 \leq n \leq l-1 .
$$


For the sake of convenience we note the values of $\triangle_{j}^{n}$ at the initial stage $n=0$. From (2.7) we have $u_{j}^{0}=\max (0, j h-E)$, and

$$
\triangle_{j}^{0}=\frac{u_{j-1}^{0}-2 u_{j}^{0}+u_{j+1}^{0}}{h^{2}}= \begin{cases}0, & \text { if } j \neq \frac{N}{2} \\ \frac{1}{h}, & \text { if } j=\frac{N}{2} .\end{cases}
$$

Thus scheme (3.5), (3.9), (3.10) can be written in vector form as

$$
\begin{gathered}
u^{n+1}=\left(I+\frac{k}{h^{2}} B(n) A\right) u^{n}, 0 \leq n \leq l-1, \\
u^{l}=\left[\prod_{n=l-1}^{0}\left(I+\frac{k}{h^{2}} B(n) A\right)\right] u^{0} .
\end{gathered}
$$

If we are interested in the computation of the numerical solution $v_{j}^{l} \approx V\left(S_{j}, t\right)$ of the original problem (1.6) at the point $\left(S_{j}, t\right)$, where from $(2.1)-(2.2)$ one gets

$$
\begin{gathered}
l k \frac{\sigma_{0}^{2}}{2}=T-t \\
v_{j}^{l}=\mathrm{e}^{-r(T-t)} u_{j}^{l}, \quad 0 \leq j \leq N,
\end{gathered}
$$

being $u_{j}^{l}$ the $j$-th component of the numerical solution of (3.17) evaluated at

$$
X_{j}=\mathrm{e}^{r(T-t)} S_{j}
$$

For the sake of clarity in the study of the properties of the numerical solution of (2.5)-(2.7) it is convenient the study of behaviour of $\triangle_{j}^{n}$ appearing in the coefficients of (3.5)-(3.6).

Let us introduce the vector

$$
\triangle^{n}=\left[\triangle_{0}^{n}, \ldots, \triangle_{N}^{n}\right]^{t}, \quad 0 \leq n \leq l-1,
$$

where $\triangle_{j}^{n}$ is given by (3.3) for the internal mesh points $1 \leq j \leq N-1$, and from (3.13), $\triangle_{0}^{n}=\triangle_{N}^{n}=0$. From (3.3) and (3.11), we can write

From (3.16) and (3.22) one gets

$$
\triangle^{n}=\frac{1}{h^{2}} A u^{n} .
$$

$$
\begin{aligned}
& \triangle^{n+1}=\frac{1}{h^{2}} A\left(I+\frac{k}{h^{2}} B(n) A\right) u^{n}=\triangle^{n}+\frac{k}{h^{2}} A B(n)\left(\frac{1}{h^{2}} A u^{n}\right)=\triangle^{n}+\frac{k}{h^{2}} A B(n) \triangle^{n}, \\
& \triangle^{n+1}=\left(I+\frac{k}{h^{2}} A B(n)\right) \triangle^{n}, 0 \leq n \leq l-1 .
\end{aligned}
$$

Componentwise expression of (3.23) takes the form

$$
\begin{aligned}
& \triangle_{j}^{n+1}=\left(1-\frac{2 k}{h^{2}} \beta_{j}^{n}\right) \triangle_{j}^{n}+\frac{k}{h^{2}} \beta_{j-1}^{n} \triangle_{j-1}^{n}+\frac{k}{h^{2}} \beta_{j+1}^{n} \triangle_{j+1}^{n}, \quad 1 \leq j \leq N-1 . \\
& \triangle_{0}^{n}=0, \quad \triangle_{N}^{n}=0, \quad 0 \leq n \leq l-1 .
\end{aligned}
$$


We conclude this section by writing the link between the action of operator $\triangle^{l}$ on the solutions of problem (1.6) and (2.5)-(2.7). From the previous comments, see (3.18)-(3.20), one gets the following approximation of the Gamma of the option:

$$
\triangle_{j}^{l}(v)=\mathrm{e}^{r(T-t)} \triangle_{j}^{l}(u), \quad 0 \leq j \leq N
$$

\section{Properties of the nUmerical SOlution}

We begin this section by writing the componentwise expression of the scheme (3.5), (3.6), (3.9), (3.10), given by

$$
\begin{aligned}
& u_{j}^{n+1}=\left(1-\frac{2 k}{h^{2}} \beta_{j}^{n}\right) u_{j}^{n}+\frac{k}{h^{2}} \beta_{j}^{n}\left(u_{j-1}^{n}+u_{j+1}^{n}\right) ; \quad 1 \leq j \leq N-1, \quad 0 \leq n \leq l-1 \\
& u_{0}^{n}=0, \quad u_{N}^{n}=X_{N}-E=E, \quad 0 \leq n \leq l \\
& u_{j}^{0}=\max (0, j h-E), \quad 0 \leq j \leq N .
\end{aligned}
$$

Note that from (3.19) the numerical solution $v_{j}^{l}$ of the original problem (1.6) is non-negative if and only if the numerical solution $u_{j}^{n}$ of (4.1) is also non-negative.

From (4.1) it is clear that assuming

$$
u_{j}^{n} \geq 0 \text { for all } j \text { with } 1 \leq j \leq N-1
$$

then it follows that

$$
u_{j}^{n+1} \geq 0 \text { for all } j \text { with } 1 \leq j \leq N-1 \text {, }
$$

if stepsizes $h, k$ satisfy

$$
\frac{k}{h^{2}} \leq \frac{1}{2 \beta_{j}^{n}}, \quad 1 \leq j \leq N-1
$$

However, condition (4.4) is not manageable because it depends on the stages $n$ and $j$. From a practical point of view, it is convenient to find a condition of the type (4.4) but independent of $j$ and $n$.

Since coefficients $\beta_{j}^{n}$ of scheme (4.1) are related to the operator $\triangle_{j}^{n}$ throughout (3.6), the following lemma studies properties of this operator whose behaviour is described by (3.24) and that will be used to show the positiveness of $u_{j}^{n}$.

Lemma 4.1. Let a be the coefficient appearing in equation (2.5), let $E$ be strike price and let $A_{2}$ and $d_{2}$ be defined by (2.10). Then, if $h=\triangle X, k=\triangle \tau$ with $N h=2 E$, satisfy the condition

$$
\frac{k}{h^{3}}\left(\left(1+d_{2}\right) h+4 a^{2} E^{2} \Psi^{\prime}\left(A_{2}\right)\right) \leq \frac{1}{8 E^{2}},
$$

the following properties hold true:

$$
\begin{aligned}
& \text { (i) } \quad \triangle_{j}^{n} \geq 0, \quad 0 \leq j \leq N, \quad 0 \leq n \leq l \\
& \text { (ii) } \quad \sum_{j=0}^{N} \triangle_{j}^{n+1} \leq \sum_{j=0}^{N} \triangle_{j}^{n}, \quad 0 \leq n \leq l-1 .
\end{aligned}
$$


Proof. Both properties are proved using the induction principle on the index $n$. For $n=0$, from (3.15) one gets that $\triangle_{j}^{0} \geq 0$ for all $j=0,1, \ldots, N$ and from (3.24) one gets

$$
\sum_{j=0}^{N} \triangle_{j}^{1}=\triangle_{\frac{N}{2}-1}^{1}+\triangle_{\frac{N}{2}}^{1}+\triangle_{\frac{N}{2}+1}^{1}=\triangle_{\frac{N}{2}}^{0}=\sum_{j=0}^{N} \triangle_{j}^{0} .
$$

Thus (ii) is proved for $n=0$.

Let us assume that properties (i) and (ii) hold true up n, i.e.,

$$
\left.\begin{array}{c}
\triangle_{j}^{n} \geq 0, \quad 0 \leq j \leq N \\
\sum_{j=0}^{N} \triangle_{j}^{0} \geq \sum_{j=0}^{N} \triangle_{j}^{1} \geq \ldots \geq \sum_{j=0}^{N} \triangle_{j}^{n} .
\end{array}\right\}
$$

Taking into account the induction hypothesis (4.6), (3.24) and that $\beta_{j}^{n} \geq 0$ by Remark 3.1 , it follows that

$$
\begin{aligned}
\sum_{j=0}^{N} \triangle_{j}^{n+1} & =\triangle_{0}^{n+1}+\sum_{j=1}^{N-1}\left(1-\frac{2 k}{h^{2}} \beta_{j}^{n}\right) \triangle_{j}^{n}+\sum_{j=0}^{N-2} \frac{k}{h^{2}} \beta_{j}^{n} \triangle_{j}^{n}+\sum_{j=2}^{N} \frac{k}{h^{2}} \beta_{j}^{n} \triangle_{j}^{n}+\triangle_{N}^{n+1} \\
& =0+\sum_{j=1}^{N-1} \triangle_{j}^{n}-\frac{k}{h^{2}} \beta_{1}^{n} \triangle_{1}^{n}-\frac{k}{h^{2}} \beta_{N-1}^{n} \triangle_{N-1}^{n}+0 \leq \sum_{j=0}^{N} \triangle_{j}^{n} .
\end{aligned}
$$

It remains to prove that $\triangle_{j}^{n+1} \geq 0$, for $1 \leq j \leq N-1$. Note that from (3.6) and using (4.6) and the monotonic increasing property of $\Psi$, see Theorem 1.1 of [4], one gets

$$
\begin{aligned}
\beta_{j}^{n} & =\left(1+\Psi\left(a^{2} X_{j}^{2} \triangle_{j}^{n}\right)\right) X_{j}^{2} \leq\left(1+\Psi\left(a^{2} X_{j}^{2}\left(\sum_{j=0}^{N} \triangle_{j}^{n}\right)\right)\right) X_{j}^{2} \\
& \leq\left(1+\Psi\left(a^{2} X_{j}^{2}\left(\sum_{j=0}^{N} \triangle_{j}^{0}\right)\right)\right) X_{j}^{2}=\left(1+\Psi\left(a^{2} X_{j}^{2} \triangle_{\frac{N}{2}}^{0}\right)\right) X_{j}^{2} .
\end{aligned}
$$

Let us denote

$$
L(h)=\frac{4 E^{2}}{h}\left(\left(1+d_{2}\right) h+4 a^{2} E^{2} \Psi^{\prime}\left(A_{2}\right)\right) .
$$

Since $X_{j} \in[0,2 E]$ and from the upper bound of $\Psi$ given by (2.11) and (4.7), (4.8) it follows that

$$
\beta_{j}^{n} \leq L(h), \quad 0 \leq j \leq N, \quad 0 \leq n \leq l .
$$

Note that condition (4.5) means that

and from (4.9), (4.10) one gets

$$
\frac{2 k}{h^{2}} L(h) \leq 1
$$

$$
1-\frac{2 k}{h^{2}} \beta_{j}^{n} \geq 0
$$

and from (4.11) and (3.24) one concludes that $\triangle_{j}^{n+1} \geq 0$. Thus the result is established. 
Note that under condition (4.5) that is independent of $n$ and $j$, all the coefficients of scheme (4.1) are non-negative, as it is clear from (4.11). The following result shows that the solution of scheme (4.1) is positive, monotonic increasing in the space-index $j$ and that the vector scheme (3.16) has a norm bounded solution.

Theorem 4.2. Let $\left\{u_{j}^{n}\right\}$ be the solution of (4.1) and let us assume condition (4.5). Then the following properties hold:

(i) $u_{j}^{n} \geq 0$, for $0 \leq j \leq N, \quad 0 \leq n \leq l$.

(ii) For $n$ fixed with $0 \leq n \leq l$, one gets

$$
u_{0}^{n} \leq u_{1}^{n} \leq \ldots \leq u_{j}^{n} \leq u_{j+1}^{n} \leq \cdots \leq u_{N}^{n} .
$$

(iii) The vector solution $\left\{u^{n}\right\}$ of system (3.16) given by (3.17) satisfies the time stability property

$$
\left\|u^{n}\right\| \leq \sqrt{6}\left\|u^{0}\right\|, \quad 1 \leq n \leq l
$$

Proof. From previous comments to the statement of Theorem 4.2, part (i) is proved. For the proof of part (ii) we use the induction principle on the index $n$. For $n=0$, from the initial condition of (4.1) one gets

$$
u_{j+1}^{0}-u_{j}^{0}=h, \quad \text { if } \quad \frac{N}{2} \leq j \leq N-1
$$

and

Thus part (ii) holds true for $n=0$. Let us assume that

$$
u_{j+1}^{0}-u_{j}^{0}=0, \quad \text { if } \quad 0 \leq j \leq \frac{N}{2}-1 .
$$

$$
u_{j+1}^{n} \geq u_{j}^{n}, \quad 0 \leq j \leq N-1 .
$$

From (4.1) and (4.12), for $1 \leq j \leq N-1,0 \leq n \leq l-1$ one gets

$$
u_{j}^{n+1}-u_{j}^{n} \leq-\frac{2 k}{h^{2}} \beta_{j}^{n} u_{j}^{n}+\frac{k}{h^{2}} \beta_{j}^{n}\left(u_{j}^{n}+u_{j+1}^{n}\right)=\frac{k}{h^{2}} \beta_{j}^{n}\left(u_{j+1}^{n}-u_{j}^{n}\right),
$$

and

Let us write

$$
u_{j}^{n+1}-u_{j}^{n} \geq-\frac{2 k}{h^{2}} \beta_{j}^{n} u_{j}^{n}+\frac{k}{h^{2}} \beta_{j}^{n}\left(u_{j-1}^{n}+u_{j}^{n}\right)=-\frac{k}{h^{2}} \beta_{j}^{n}\left(u_{j}^{n}-u_{j-1}^{n}\right) .
$$

$$
u_{j+1}^{n+1}-u_{j}^{n+1}=\left(u_{j+1}^{n+1}-u_{j+1}^{n}\right)+\left(u_{j+1}^{n}-u_{j}^{n}\right)-\left(u_{j}^{n+1}-u_{j}^{n}\right) .
$$

From (4.13)-(4.15) and (4.9) and (4.10) it follows that

$$
\begin{aligned}
u_{j+1}^{n+1}-u_{j}^{n+1} & \geq-\frac{k}{h^{2}} \beta_{j+1}^{n}\left(u_{j+1}^{n}-u_{j}^{n}\right)+\left(u_{j+1}^{n}-u_{j}^{n}\right)-\frac{k}{h^{2}} \beta_{j}^{n}\left(u_{j+1}^{n}-u_{j}^{n}\right) \\
& =\left(1-\frac{k}{h^{2}}\left(\beta_{j+1}^{n}+\beta_{j}^{n}\right)\right)\left(u_{j+1}^{n}-u_{j}^{n}\right) \\
& \geq\left(1-\frac{k}{h^{2}} 2 L(h)\right)\left(u_{j+1}^{n}-u_{j}^{n}\right) \geq 0 .
\end{aligned}
$$


This proves (ii) for $1 \leq j \leq N-2$. For $j=0$ we have $u_{0}^{n}=0 \leq u_{1}^{n}$ by part (i) and from (4.15) for $j=N-1$ and the induction hypothesis one gets

$$
u_{N}^{n+1}-u_{N-1}^{n+1} \geq\left(1-\frac{k}{h^{2}} \beta_{N-1}^{n}\right)\left(u_{N}^{n}-u_{N-1}^{n}\right) \geq\left(1-\frac{2 k}{h^{2}} L(h)\right)\left(u_{N}^{n}-u_{N-1}^{n}\right) \geq 0 .
$$

Thus part (ii) is established.

In order to prove (iii) note that from (ii) is follows that

$$
u_{0}^{n}=0 \leq u_{j}^{n} \leq u_{N}^{n}=E, \quad 1 \leq j \leq N-1
$$

From (4.16) one gets

$$
\left\|u^{n}\right\|^{2}=\left\|\left(u_{0}^{n}, u_{1}^{n}, \ldots, u_{N}^{n}\right)^{t}\right\|^{2} \leq\left\|E(0,1,1, \ldots, 1)^{t}\right\|^{2}=N E^{2} .
$$

Note that $u^{0}=\left[0,0, \ldots, 0, h, 2 h, \ldots, \frac{N}{2} h\right]^{t}$, where the first nonzero component corresponds to the position $\frac{N}{2}+1$, and thus

$$
\left\|u^{0}\right\|^{2}=h^{2}\left(\sum_{j=1}^{\frac{N}{2}} j^{2}\right)=h^{2} \frac{N(N+1)(N+2)}{24} .
$$

Taking into account that $N=\frac{2 E}{h}$, from (4.17), (4.18) it follows that

$$
\frac{\left\|u^{n}\right\|}{\left\|u^{0}\right\|} \leq \frac{2 E \sqrt{6}}{N h}=\sqrt{6}
$$

Thus part (iii) is established.

\section{NumERICAL ANALYSIS AND FINANCIAL INTERPRETATION}

With the notation of Section 3, the numerical solution $v_{j}^{l}$ of problem (1.6) at the point $\left(S_{j}, t\right)$ is given by

$$
v_{j}^{l}=\mathrm{e}^{-r(T-t)} u_{j}^{l}, \quad X_{j}=\mathrm{e}^{r(T-t)} S_{j}
$$

From Theorem 4.2 and (5.1) the following result holds true and guarantees that the option pricing does not has spurious oscillations under condition (4.5).

Corollary 5.1. With previous notation and under condition (4.5) the option pricing numerical solution $v_{j}^{l}$ of problem (1.6) at $\left(S_{j}, t\right)$ is nonnegative and monotonic increasing with the underlying asset $S_{j}$ at a fixed time $t$.

The stability of the numerical solution $\left\{u^{n}\right\}$ of scheme (3.16) guarantees that option pricing at time $t$ is bounded by a quantity which depends on the remaining time to maturity $T-t$. Note that from (5.1) and part (ii) of Theorem 4.2 , for $S_{j} \in[0,2 E], 0 \leq j \leq N$, one gets that $v_{j}^{l} \approx V\left(S_{j}, t\right)$ satisfies

$$
v_{j}^{l}=\mathrm{e}^{-r(T-t)} u_{j}^{l} \leq \mathrm{e}^{-r(T-t)} u_{N}^{l}=\mathrm{e}^{-r(T-t)}\left(X_{N}-E\right)=\mathrm{e}^{-r(T-t)}\left(\mathrm{e}^{r(T-t)} 2 E-E\right)=\left(2-\mathrm{e}^{-r(T-t)}\right) E .
$$

The next result shows that numerical scheme (4.1) is consistent with equation (2.5)-(2.7). Consistency means that the exact theoretical solution of the partial differential equation approximates well to the exact solution of scheme as the stepsizes tends to 0 [17]. This property is important for the reliability of the numerical solution because a bad numerical solution may waste a good mathematical model. 
Let $F\left(u_{j}^{n}\right)=0$ represent the approximating difference equation defined by

$$
F\left(u_{j}^{n}\right)=\frac{u_{j}^{n+1}-u_{j}^{n}}{k}+\frac{2}{h^{2}} \beta_{j}^{n} u_{j}^{n}-\frac{\beta_{j}^{n}}{h^{2}}\left(u_{j-1}^{n}+u_{j+1}^{n}\right)=0, \quad 1 \leq j \leq N-1 .
$$

In accordance with [17], p. 100, the scheme (4.1) is consistent with (2.5) if

$$
T_{j}^{n}(U)=F\left(U_{j}^{n}\right)-L\left(U_{j}^{n}\right) \rightarrow 0, \text { as } h=\triangle X \rightarrow 0, \quad k=\triangle \tau \rightarrow 0
$$

where $U_{j}^{n}$ denotes the theoretical solution of (2.5) evaluated at $\left(X_{j}, \tau^{n}\right)$.

Using operator $\triangle_{j}^{n}$ introduced in (3.3), let us write

$$
F\left(U_{j}^{n}\right)=\frac{U_{j}^{n+1}-U_{j}^{n}}{k}-\beta_{j}^{n}(U) \triangle_{j}^{n}(U)
$$

Assuming that $U$ is four times continuously differentiable with respect to $X$ and using Taylor expansion about $\left(X_{j}, \tau^{n}\right)$ it follows that

$$
\triangle_{j}^{n}(U)=\frac{\partial^{2} U}{\partial X^{2}}\left(X_{j}, \tau^{n}\right)+\frac{h^{2}}{12} \frac{\partial^{4} U}{\partial X^{4}}\left(\eta, \tau^{n}\right)=\frac{\partial^{2} U}{\partial X^{2}}\left(X_{j}, \tau^{n}\right)+h^{2} E_{j}^{n}(1), \quad X_{j}-h<\eta<X_{j}+h
$$

where

$$
\left|E_{j}^{n}(1)\right| \leq \frac{1}{12} \max \left\{\left|\frac{\partial^{4} U}{\partial X^{4}}\left(X, \tau^{n}\right)\right| ; \quad 0 \leq X \leq X_{N}=2 E\right\}=\frac{\left|U^{n}(1)\right|_{\max }}{12}
$$

Assuming that $U$ admits two times continuous partial derivatives with respect to $\tau$, it follows that

$$
\frac{U_{j}^{n+1}-U_{j}^{n}}{k}=\frac{\partial U}{\partial \tau}\left(X_{j}, \tau^{n}\right)+k E_{j}^{n}(2)
$$

where

$$
\begin{gathered}
E_{j}^{n}(2)=\frac{1}{2} \frac{\partial^{2} U}{\partial \tau^{2}}\left(X_{j}, \delta\right), \quad \tau^{n}=n k<\delta<\tau^{n+1}=(n+1) k \\
\left|E_{j}^{n}(2)\right| \leq \frac{1}{2}\left|U_{j}^{n}(2)\right|_{\max }=\frac{1}{2} \max \left\{\left|\frac{\partial^{2} U}{\partial \tau^{2}}\left(X_{j}, \tau\right)\right| ; \quad \tau^{n} \leq \tau \leq \tau^{n+1}\right\} .
\end{gathered}
$$

From (5.4), (5.5) and (5.7) one gets

$$
\begin{aligned}
T_{j}^{n}(U) & =F\left(U_{j}^{n}\right)-L\left(U_{j}^{n}\right) \\
& =-X_{j}^{2}\left\{\left(1+\Psi_{j}^{n}(U)\right) \triangle_{j}^{n}(U)-\left(1+\Psi\left(a^{2} X_{j}^{2} \frac{\partial^{2} U}{\partial X^{2}}\left(X_{j}, \tau^{n}\right)\right)\right) \frac{\partial^{2} U}{\partial X^{2}}\left(X_{j}, \tau^{n}\right)\right\}+k E_{j}^{n}(2),
\end{aligned}
$$

where

$$
\Psi_{j}^{n}(U)=\Psi\left(a^{2} X_{j}^{2} \triangle_{j}^{n}(U)\right)
$$

Let us introduce the notation

$$
A_{j}^{n}=a^{2} X_{j}^{2} \frac{\partial^{2} U}{\partial X^{2}}\left(X_{j}, \tau^{n}\right) ; \quad \triangle A_{j}^{n}=a^{2} X_{j}^{2} E_{j}^{n}(1) h^{2}
$$




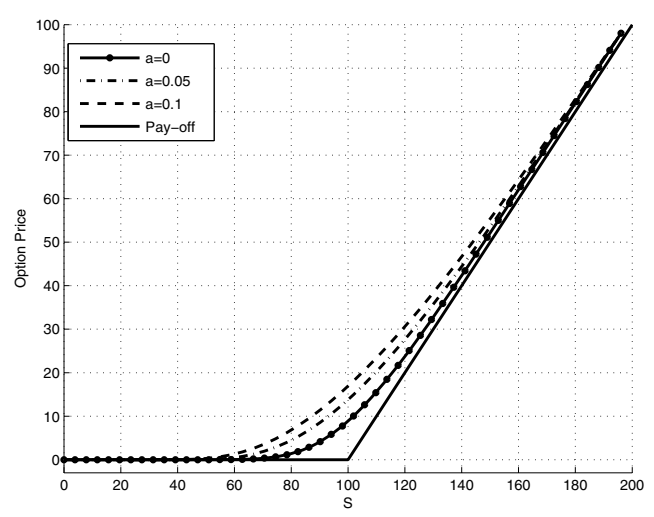

FIGURE 1. Option pricing for several values of parameter $a$ at time to maturity 1 year.

From (5.12) and using function $g(A)$ introduced in Lemma 2.1 one gets

$$
\begin{aligned}
& \left\{\left(1+\Psi_{j}^{n}(U)\right) \triangle_{j}^{n}(U)-\left(1+\Psi\left(a^{2} X_{j}^{2} \frac{\partial^{2} U}{\partial X^{2}}\left(X_{j}, \tau^{n}\right)\right)\right) \frac{\partial^{2} U}{\partial X^{2}}\left(X_{j}, \tau^{n}\right)\right\} \\
& =\left\{\left(1+\Psi\left(A_{j}^{n}+\triangle A_{j}^{n}\right)\right)\left(A_{j}^{n}+\triangle A_{j}^{n}\right)-\left(1+\Psi\left(A_{j}^{n}\right)\right) A_{j}^{n}\right\} a^{-2} X_{j}^{-2} \\
& =\left\{g\left(A_{j}^{n}+\triangle_{j}^{n}\right)-g\left(A_{j}^{n}\right)+\triangle A_{j}^{n}\right\} a^{-2} X_{j}^{-2} .
\end{aligned}
$$

From $(5.10),(5.13)$ and the differentiability of $g(A)$ given in Lemma 2.1 it follows that

$$
T_{j}^{n}(U)=-X_{j}^{2}\left(1+g^{\prime}\left(A_{j}^{n}+\theta\left(\triangle A_{j}^{n}\right)\right)\right) E_{j}^{n}(1) h^{2}+k E_{j}^{n}(2),
$$

for some $\theta$ with $0<\theta<1$.

From equation (2.12) of Lemma 2.1 together with (5.6), (5.9) and (5.14), it follows that

$$
\left|T_{j}^{n}(U)\right| \leq \frac{h^{2} E^{2}}{3}(1+C(n, h))\left|U^{n}(1)\right|_{\max }+\frac{k}{2}\left|U_{j}^{n}(2)\right|_{\max }
$$

where

$$
\begin{gathered}
C(n, h)=\max \left\{G, 8 a^{2} E^{2}\left(\frac{h^{2}}{12}\left|U^{n}(1)\right|_{\max }+\left|U^{n}(3)\right|_{\max } \Psi^{\prime}\left(A_{2}\right)+d_{2}\right)\right\}, \\
\left|U^{n}(3)\right|_{\max }=\max \left\{\left|\frac{\partial^{2} U}{\partial X^{2}}\left(X_{j}^{2}, \tau^{n}\right)\right| ; \quad 0 \leq X_{j} \leq 2 E\right\} .
\end{gathered}
$$

Summarizing, from (5.15)-(5.17) one gets

$$
T_{j}^{n}(U)=O\left(h^{2}\right)+O(k)
$$

and the following result has been established:

Theorem 5.2. Assuming that the exact solution of (2.5)-(2.7) admits four times continuous partial derivatives with respect to $X$ and two times continuous partial derivatives with respect to $\tau$, the scheme (4.1) is consistent with (2.5) and behaves $T_{j}^{n}(U)=O\left(h^{2}\right)+O(k)$ as $h \rightarrow 0, k \rightarrow 0$. 


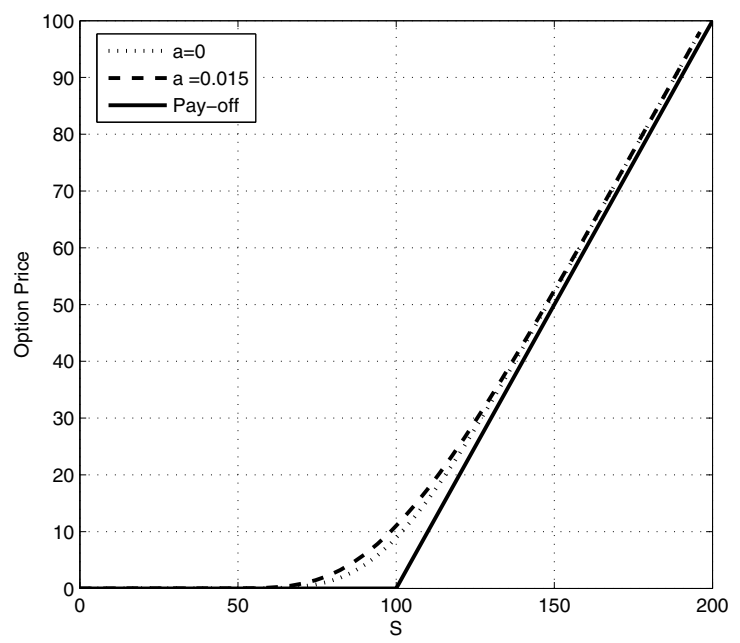

FIGURE 2. Stable option pricing for $h=4, k=3.2787 \times 10^{-5}$.

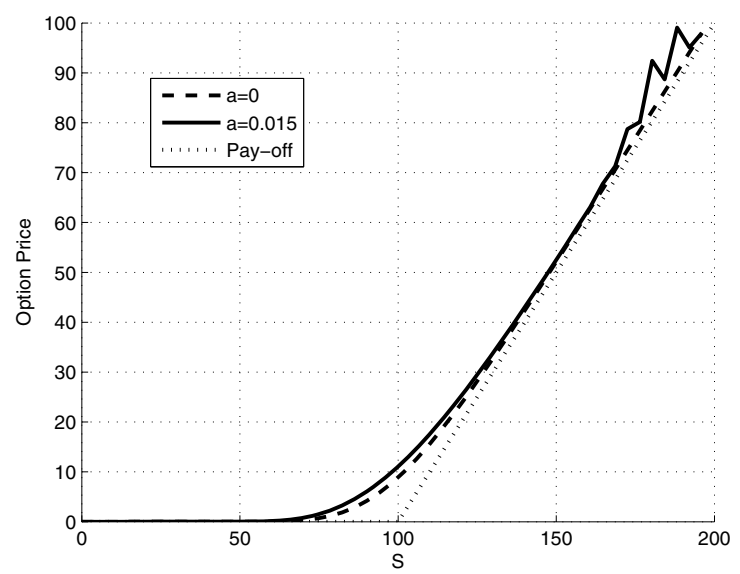

FIGURE 3. Wrong option pricing for $h=4, k=2.7027 \times 10^{-4}$.

\section{ExAmples}

All the computations of the numerical solutions are performed using the scheme for the transformed problem (2.5)-(2.7) and further translation to the option price of the original problem (1.6), using (3.19).

The first example shows that the price of an European vanilla call option increases with the parameter $a$.

Example 6.1. Consider the option pricing of an European vanilla call option with data

$$
\begin{gathered}
E=100 ; \quad \sigma_{0}=0.2 ; \quad r=0.02 ; \quad T-t=1 \text { year } \\
h=\triangle X=4 ; \quad k=\Delta \tau=1.7606 \times 10^{-6} .
\end{gathered}
$$

Figure 1 shows the numerical solution for different values of the parameter $a$, as well as the Black-Scholes solution for $a=0$. 


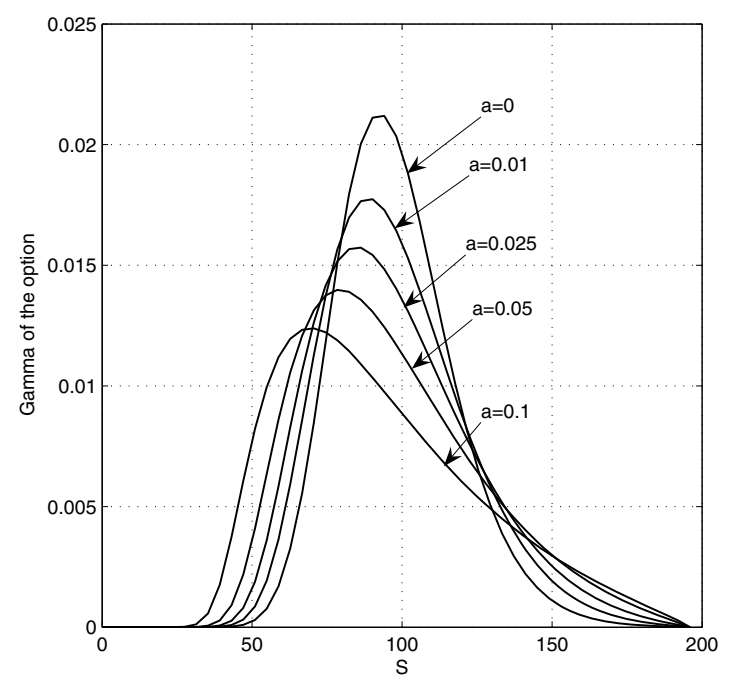

FiguRE 4. Numerical values of the Gamma of the option for different values of $a$.

Remark 6.2. Note that parameter $a$ has a direct influence in the stability condition (4.5). In particular, for a fixed value of $h$, higher values of the parameter $a$ imposes smaller values of the stepsize $k$.

The next example shows a situation where the numerical solution behaves well under the stability condition (4.5) and also that if such condition is not satisfied, then they appear spurious oscillations.

Example 6.3. With the same data as Example 6.1 with $a=0.015$ and with several stepsizes for $k$ and fixed value $h=4$, Figure 2 shows the good behaviour of the stable numerical solution and Figure 3 shows the wrong numerical solution when the stability condition is not satisfied.

The following example shows the numerical value of the Gamma of an European vanilla call option showing that the maximum of the quantity is achieved on the left of the strike price as the value of the parameter $a$ increases and that the maximum decreases with the value of $a$.

Example 6.4. With the data of Example 6.1, Figure 4 shows the numerical values of Gamma obtained from (3.24) and (3.25).

\section{REFERENCES}

[1] M. Avellaneda and A. Parás, Dynamic hedging portfolios for derivative securities in the presence of large transaction costs. Appl. Math. Finance 1 (1994) 165-193.

[2] G. Barles and H.M. Soner, Option pricing with transaction costs and a nonlinear Black-Scholes equation. Finance Stochast. 2 (1998) 369-397.

[3] P. Boyle and T. Vorst, Option replication in discrete time with transaction costs. J. Finance 47 (1973) 271-293.

[4] R. Company, E. Navarro, J.R. Pintos and E. Ponsoda, Numerical solution of linear and nonlinear Black-Scholes option pricing equations. Comput. Math. Appl. 56 (2008) 813-821.

[5] M. Davis, V. Panis and T. Zariphopoulou, European option pricing with transaction fees. SIAM J. Contr. Optim. 31 (1993) 470-493.

[6] J. Dewynne, S. Howinson and P. Wilmott, Option pricing: mathematical models and computations. Oxford Financial Press, Oxford (2000).

[7] B. Düring, M. Fournier and A. Jungel, Convergence of a high order compact finite difference scheme for a nonlinear BlackScholes equation. ESAIM: M2AN 38 (2004) 359-369.

[8] P. Forsyth, K. Vetzal and R. Zvan, A finite element approach to the pricing of discrete lookbacks with stochastic volatility. Appl. Math. Finance 6 (1999) 87-106. 
[9] J.M. Harrison and S.R. Pliska, Martingales and stochastic integrals in the theory of continuous trading. Stochastic Processes Appl. 11 (1981) 215-260.

[10] S.D. Hodges and A. Neuberger, Optimal replication of contingent claims under transaction costs. Review of Futures Markets 8 (1989) 222-239.

[11] T. Hoggard, A.E. Whalley and P. Wilmott, Hedging option portfolios in the presence of transaction costs. Adv. Futures Options Research 7 (1994) 217-35.

[12] R. Kangro and R. Nicolaides, Far field boundary conditions for Black-Scholes equations. SIAM J. Numer. Anal. 38 (2000) $1357-1368$.

[13] J. Leitner, Continuous time CAPM, price for risk and utility maximization, in Mathematical Finance - Workshop of the Mathematical Finance Research Project, Konstanz, Germany, M. Kohlmann and S. Tang Eds., Birkhäuser, Basel (2001).

[14] H.E. Leland, Option pricing and replication with transactions costs. J. Finance 40 (1985) 1283-1301.

[15] O. Pironneau and F. Hecht, Mesh adaption for the Black and Scholes equations. East-West J. Numer. Math. 8 (2000) 25-35.

[16] A. Rigal, Numerical analisys of three-time-level finite difference schemes for unsteady diffusion-convection problems. J. Num. Meth. Engineering 30 (1990) 307-330.

[17] G.D. Smith, Numerical solution of partial differential equations: finite difference methods. Third Edition, Clarendon Press, Oxford (1985).

[18] H.M. Soner, S.E. Shreve and J. Cvitanic, There is no non-trivial hedging portfolio for option pricing with transaction costs. Ann. Appl. Probab. 5 (1995) 327-355.

[19] J.C. Strikwerda, Finite difference schemes and partial differential equations. Wadsworth \& Brooks/Cole Mathematics Series (1989) $32-52$.

[20] D. Tavella and C. Randall, Pricing financial instruments - The finite difference method. John Wiley \& Sons, Inc., New York (2000).

[21] A.E. Whalley and P. Wilmott, An asymptotic analysis of an optimal hedging model for option pricing with transaction costs. Math. Finance 7 (1997) 307-324. 